\title{
The need for a prescribing competency framework to address the burden of complex polypharmacy among multiple long-term conditions
}

\author{
Authors: Catherine Picton, ${ }^{\mathrm{A}}$ Claire Loughrey ${ }^{\mathrm{B}}$ and Andrew Webb ${ }^{\mathrm{C}}$
}

The original requirement for a competency framework for prescribers was to identify the essential skills for nonmedical prescribers. However, core prescribing competencies are relevant to any prescriber. The new, revised version is especially relevant for doctors/physicians. Doctors are the most frequent prescribers - prescribing is the most common therapeutic intervention. The quantity and complexity of medicine use is increasing through multiple treatment strategies/pathways for multiple comorbidities, resulting in polypharmacy - especially with long-term conditions. This is against a background of the ongoing introduction of new drugs with novel mechanisms of action with increased risks of adverse effects, compounded by drug-drug and diseasedrug interactions. This has increased the need for monitoring and follow-up, including identification and management of poor adherence. It is challenging for doctors to maintain safe and effective prescribing and train other doctors and non-medical prescribers within the multidisciplinary team.

The prescribing competency framework provides a systematic approach to support doctors to prescribe safely and effectively. It can be used by medical schools to teach prescribing, including preparation for the prescribing safety assessment; by F1/F2 doctors to support prescribing in early years; as part of prescribing quality improvement initiatives and as a continuing professional development framework in general practice or acute care settings.

\section{The need for a prescribing competency framework}

Increasing complexity of prescribing

Medicines are used more than any other intervention to manage medical conditions. Both the number of medicines prescribed and the complexity of the medicine regimens that patients take are increasing. As the population ages and

Authors: ${ }^{\text {A }}$ consultant, Royal Pharmaceutical Society, London, UK; ${ }^{B}$ director of postgraduate general practice education, Northern Ireland Medical and Dental Training Agency, Belfast, UK; ${ }^{\text {C senior }}$ lecturer and honorary consultant, King's College London, London, UK multiple comorbidities become more prevalent, polypharmacy is increasingly becoming the norm for patients. ${ }^{1}$ A Royal College of General Practitioners (RCGP) review of the workload in general practice highlighted the 19\% increase in GP consultations in England between 2008-09 and 2013-14 and the increased complexity of those patients presenting, citing the number of patients with comorbidities are set to rise by $53 \%$ between 2008 and 2018. ${ }^{2}$ A review of GP workload in Northern Ireland last year demonstrated that, in 2013, 39 million prescription items were prescribed - approximately 20 items per patient per year. ${ }^{3}$

This increase in complexity means that besides developing and maintaining prescribing competency for individual conditions, prescribers have the challenge of keeping up to date with new medicines as they come on the market and being aware of the potential for interaction between medicines in patients with multiple comorbidities. ${ }^{1}$

\section{Key points}

Prescribing safely and effectively is a challenge, particularly when prescribing for people with multiple long-term conditions and complex polypharmacy.

Doctors will continue to be challenged as new medicines are introduced with novel mechanisms of action, adverse effects and drug-drug or disease-drug interactions.

The need to understand how patients use medicines in practice is increasingly being recognised and requires more monitoring and follow-up, including a focus on adherence.

A core set of prescribing competencies relevant to all prescribers has been updated and can be used to underpin safe and effective prescribing.

The competency framework can be used by medical schools to support doctors in training and by all doctors at any stage in their career, as well as helping doctors supporting non-medical prescribers.

KEYWORDS: Competency framework, prescriber, prescribing and competency 
Specialists in hospitals are now often faced with patients taking medicines for two or three long-term conditions in addition to the one that they are treating. This shift is reflected in the debate about the increasing need for generalist medical skills in hospitals so that there are doctors able to coordinate the care of patients with complex needs. ${ }^{4}$ In fact, the trend towards increased specialisation within medical training has recently come under scrutiny, not least in the Shape of training review. ${ }^{5}$ This report makes the case for more generalist training for all doctors to meet patient needs.

Similarly, in general practice, it has been recognised that GPs need to enhance their expert generalist clinical skills but also need to develop additional skills for extended roles. ${ }^{6}$ The need to ensure that patients' medicines are regularly reviewed, particularly those with multiple comorbidities, has driven the debate about consultation times and the effective use of the multidisciplinary team skill mix (including other prescribing professions such as nurses and pharmacists) to ensure that this happens. ${ }^{7}$

\section{Clear need to optimise the use of medicines in practice}

Effective use of medicines is often focused on getting the choice of medicine right for an individual patient. While this has the potential to improve quality of life and patient outcomes,

\section{Box 1. Are we making the most of medicines?}

\section{Do patients take their medicines?}

Only $16 \%$ of patients who are prescribed a new medicine take it as prescribed, experience no problems and receive as much information as they need. ${ }^{9}$

> Ten days after starting a medicine, almost a third of patients are already non-adherent - of these, 55\% don't realise they are not taking their medicines correctly, while $45 \%$ are intentionally non-adherent. ${ }^{9}$

\section{How well do we use medicines?}

A study conducted in care homes found that over two thirds of residents were exposed to one or more medication errors. ${ }^{10}$

> Over half a million medication incidents were reported to the National Patient Safety Agency between 2005 and 2010: $16 \%$ of them involved actual patient harm. ${ }^{11}$

$>$ In hospitals, the General Medical Council's EQUIP study demonstrates a prescribing error rate of almost $9 \% .{ }^{12}$

In general practice, an estimated 1.7 million serious prescribing errors occurred in 2010. ${ }^{13}$

Is the NHS getting the best value from medicines?

In primary care, around $£ 300$ million of medicines are wasted per year (this is likely to be a conservative estimate), of which $€ 150$ million is avoidable. ${ }^{14}$

At least $6 \%$ of emergency re-admissions are caused by avoidable adverse reactions to medicines. ${ }^{15}$

\section{Are patients getting the right medicines?}

> Analysis of the NHS Atlas of Variation highlights unwarranted variations in the prescribing of some medicines across England.

Taken from Medicines optimisation: helping patients make the most of their medicines. ${ }^{8}$ how the medicines are used in practice is equally important. However, there are a range of problems with this ${ }^{8}$ (Box 1). ${ }^{9-15}$ Optimising the way that medicines are used is a priority not just for the UK but for many developed healthcare systems. This level of priority is reflected by the fact that the National Institute for Health and Care Excellence (NICE) has developed a guideline on medicines optimisation, which makes recommendations for how doctors, pharmacists and other members of the multiprofessional team can improve the way medicines are used. ${ }^{16}$

Poor adherence (whether intentional or unintentional) is an example of where medicines use can go wrong in practice. Poor adherence has been shown to be alarmingly prevalent in patients starting a new medicine with one study reporting that 10 days after first starting a medicine, almost a third of patients are already non-adherent. Moreover, while $45 \%$ of these patients are intentionally non-adherent, 55\% don't actually realise that they are not taking their medicines correctly. ${ }^{9}$ Similarly, a quarter of patients attending a specialist hypertension centre in Leicester for suboptimal blood pressure control apparently due to 'resistant' hypertension were totally or partially non-adherent to their antihypertensive treatment (approximately 10\% with total and $15 \%$ with partial non-adherence). This audit was a 'game-changer' in the approach to resistant hypertension: awareness of such high prevalence of non-adherence can help identify the problem in a given patient and avoid unnecessary treatment escalation. ${ }^{17}$

The potential risks to patient safety were set out by the Institute of Medicine in $1999 .{ }^{18}$ In 2002, Wilson and Sheikh considered public safety in primary care and identified prescribing as one of four key safety issues. ${ }^{19}$ They proposed that ' $3-5 \%$ of all prescriptions in primary care might cause problems and one third of these can be classified as serious'. In the PRACtICe (prevalence and causes of prescribing errors in general practice) study, which looked at errors in general practice, a wide range of underlying causes of error were identified relating to the prescriber, the patient, the team, the working environment, the task, the computer system and the primary/secondary care interface. ${ }^{13}$ As prescribing is recognised as an area of potential risk to patients, the report recommended a review of the RCGP's curriculum. ${ }^{13}$

Prescribing errors may be more prevalent in the hospital setting than primary care. The EQUIP study found a mean error rate of 8.9 errors per 100 medication orders in hospital and identified targets for intervention to reduce prescribing errors; these included undergraduate medical education programmes, foundation year 1 education, and multiprofessional education, as well as clinical working environments. ${ }^{12}$

While 'high-risk' medications, such as warfarin and disease modifying anti-rheumatic drugs (DMARDs), present a particular threat to patient safety, this risk is often preventable. This was highlighted in a systematic review whereby the incidence of preventable medicines-related admissions to hospital was $3.7 \%$ (median; range $1.4-15.4 \%$ ). ${ }^{20}$ Importantly, $50 \%$ of all such preventable drug-related admissions were linked to just four groups of medicines: antiplatelets, diuretics, nonsteroidal antiinflammatory drugs (NSAIDs) and anticoagulants.

\section{Changing patient pathways and care models}

It is not only the medicines prescribed that are increasing in number and complexity, the pathways that patients follow are 
also evolving. In England, the Five Year Forward View ${ }^{21}$ proposed the development of radically different models of delivering care that are now being realised through its Vanguard programme. There are currently 50 vanguard sites, each taking the lead on the development of new care models that will act as a blueprint for the NHS. ${ }^{22}$ The Vanguards are active across all sectors of care and are looking at new approaches to the coordination of urgent and emergency care, enhanced health in care homes, moving specialist care out of hospitals into the community, joining up GP, hospital, community and mental health services, and acute care collaborations. We are already starting to see the lines between hospital and primary care blurring, with patients previously managed in a hospital setting either transferred for ongoing care to community or primary care, or treated there in the first instance. We have also seen hospitals outsourcing ongoing medicines supplies and, in some cases, ongoing care to homecare companies; this ranges from low tech through to high tech and complex medicines regimens. ${ }^{23}$

What this means for prescribers is that the patient's care and, with it, the prescribing of medicines will move through a range of different - and in some cases new - settings. This is already having an impact on the systems and governance around prescribing and, therefore, the competencies that are associated with managing the systems aspects of prescribing should not be underestimated.

\section{More professions prescribing}

This shift to new models of care delivery is in some respects being supported by the changes to legislation made in the last 15 years to enable professions other than doctors and dentists to prescribe. This has allowed the health service to make use of the skills of these professionals to support the delivery of services. While the numbers of these prescribers are small relative to doctors, they are increasing throughout the health service across a wide range of roles, which means that doctors may increasingly be working alongside other professionals able to prescribe in their own right.

After successful completion of an approved education programme, nurses, pharmacists, optometrists, physiotherapists, podiatrists and therapeutic radiographers can prescribe independently within their own areas of competence; there are few restrictions on the medicines that they can prescribe. Regulatory standards underpin prescribing by these professional groups and, once qualified, prescribers must record their qualification with their professional regulator and have a responsibility to remain up to date with the knowledge and skills that enable them to prescribe competently and safely. As well as prescribing independently, these professionals (plus dieticians and diagnostic radiographers) can enter into a supplementary prescribing agreement with a doctor for an individual patient. This requires the development of a clinical management plan that sets the scope of what the supplementary prescriber can prescribe and when referral back to the doctor is necessary.

\section{How a competency framework can help}

The prescribing competencies that doctors should expect to develop and maintain extend beyond the identification of an appropriate medicine for an individual patient. Prescribing is multifaceted and as much about managing systems risk and the way patients use their medicines in practice as it is about clinical pharmacology - as identified by the analysis of causes of errors in general practice in the PRACtice study. ${ }^{13}$

A generic prescribing competency framework can support doctors to develop and maintain a holistic set of competencies that are patient-centred and frame prescribing in the wider context. By providing a single competency framework, there is the opportunity to bring doctors and other prescribing professionals together and to harmonise education for prescribers.

\section{How doctors use the framework}

\section{Teaching prescribing to undergraduates}

Experience from the other professions has shown that the framework is a practical and effective way to underpin the teaching of prescribing. The framework has been used widely by higher education institutes to provide a basis for developing teaching programmes and as a portfolio to demonstrate attainment of competencies (Box 2).

The framework can be mapped to existing teaching in medical schools to ensure that all relevant areas of prescribing are

\section{Box 2. Using the competency framework to train} prescribers

The Centre for Professional Development and Lifelong Learning at Keele University has been offering courses for trainee pharmacist prescribers since 2003 . Throughout the last 13 years, the competency frameworks relating to prescribing have been used both to underpin the education of the trainee prescribers and for assessment purposes.

Key to delivery of the independent prescribing course has been the development of a 'learning in practice/prescribing competency workbook'. The workbook is structured so that the course learning outcomes and prescribing competencies are linked. This workbook requires the student to document, through three separate review meetings with their designated medical practitioner (DMP), how they are working towards (in the initial and intermediate meetings) gaining competence in their specialist area of prescribing. At the final review meeting, the student needs to demonstrate that they are competent to prescribe by providing evidence for all of the course learning outcomes and prescribing competencies.

The students at Keele University use a structured reflective portfolio to collect evidence of their competence of the behavioural indicators that underpin the overarching prescribing competences. They are encouraged to write regularly in their portfolio, reflecting on the learning they have gained during their period of study. This could include learning from study days, directed reading, observation of other prescribers, direct patient care observed by others, assessment - such as case presentations and Objective Structured Clinical Examinations (OSCE) - or any other source that provides evidence of meeting the prescribing competency standards. By the end of the study period, the student is required to map all of their evidence within the portfolio against the 16 course learning outcomes and nine prescribing competencies. This is submitted to the university and assessed by tutors who are also prescribers.

Students must demonstrate their competence to prescribe by meeting all of the competencies described in the framework before they are allowed to register as prescribers. 
covered. It is consistent with the British Pharmaceutical Society curriculum in clinical pharmacology and prescribing for medical students. ${ }^{24}$ It offers medical schools that are co-located with higher education institutions providing postgraduate training in prescribing for other professions a framework to develop multidisciplinary education sessions with them. Taking this multiprofessional approach will help doctors and, in particular, junior doctors to understand that other professional groups have prescribing competencies.

Using the framework in this way will support the recommendations of the EQUIP study, which highlighted the importance of undergraduate education and multidisciplinary education as ways to reduce prescribing errors. ${ }^{12}$

\section{Supporting foundation doctors}

The Prescribing Safety Assessment ${ }^{25}$ maps across to the competency framework and provides a basis for supporting doctors in their early years of practice. It may be particularly useful, along with structured support sessions, for those doctors who need additional support with prescribing at the beginning of their career, including doctors who may have registered outside of the UK.

\section{Supporting quality improvement}

The Scottish Patient Safety Programme in Primary care (SPSP-PC) has recognised the risk that prescribing errors pose to patient safety and has put together a number of resources, including medicines management across the primary secondary care interface. ${ }^{26} \mathrm{~A}$ focus on high-risk medicines, as demonstrated by the SPSP-PC initiative, has the potential to significantly reduce patient harm. It follows that the prescribing competency framework is therefore a useful tool for doctors to apply in quality improvement for prescribing.

It could be argued that focusing attention on improvement in prescribing practice in primary care would reap significant benefits. The prescribing competency framework provides a touchstone for primary care practitioners to review their prescribing practices and inform their continuing professional development. There is a real opportunity for GP training programmes to actively endorse the prescribing competency framework to ensure that GP trainees entering the GP workforce are fully aware of the risks when prescribing in primary care and understand the potential impact of human factors and complex prescribing systems.

\section{Continuing professional development}

Perhaps the most significant strength of the competency framework is that it provides a tool around which to structure ongoing continuing professional development in prescribing that stays with doctors as their practice develops. This is because it is a generic framework that focuses on core competencies and can be used over time as doctors develop their generalist skills and/or specialise. It is the generic nature of the framework and the need to apply it to a prescribers scope of practice that make it suitable for use by anyone prescribing, regardless of their professional background.

The framework has already been used extensively across the NHS by professional groups (other than doctors) as part of governance processes, such as reaccreditation or revalidation, to ensure that prescribers remain competent to prescribe over time. In general practice, it has been used for critical case analysis and can support a multidisciplinary or team approach to continuing professional development in prescribing. It has also been used to support return to prescribing practice after a period of absence.

The General Medical Council and the Academy of Medical Royal Colleges have recently completed a consultation on generic professional capabilities for all doctors. ${ }^{27}$ The ethos of this proposal is that such capabilities should be integrated 'into all aspects of medical education and practice'. The inclusion of specific content on prescribing medicines safely highlights that safe prescribing is recognised as a core generic professional capability for doctors, reflecting the content of the prescribing competency framework.

The generic professional capabilities consultation also included many aspects referred to in the prescribing competency framework, such as communication, leadership and teamwork, patient safety and quality improvement, and dealing with complexity and uncertainty. The fact that two educational frameworks coalesce in such a way, having consulted widely and independently on each, endorses the direction of travel for both.

\section{The structure of the competency framework}

The competency framework consists of ten competencies. ${ }^{28}$ Each competency is supported by a list of indicators that describe how prescribers with that competency will be working. A key feature of this framework is that it is generic, so each prescriber will need to contextualise the statement for their individual prescribing practice. This might be for a specific patient group, eg children, or a specific category of medicines, eg for chemotherapy. The emphasis of the framework is in the development of skills rather than as a checklist exercise.

The prescribing framework reflects the multifaceted nature of prescribing. The ten competencies (Fig 1) are split into two areas: the consultation and prescribing governance. Each of the competencies is supported by statements that describe the activity or outcomes that prescribers should be able to demonstrate.

\section{Conclusion}

There is an increasing challenge to doctors when prescribing, particularly for patients with multiple long-term conditions. There is a tension between the delivery of holistic and patientcentred care and the application of evidence-based medicine. The trend towards increased specialisation within medical training is now being questioned and the case for more generalist training for all doctors to meet patient needs is increasingly being made.

The prescribing competency framework recognises this tension by presenting the framework in two sections - the consultation section, with a focus on the individual patient, and the prescribing governance section.

While doctors prescribe as a frequent patient intervention, the extension of prescribing skills to other healthcare professionals means this skill is no longer the prevail of one professional group. There is the potential for interprofessional learning and the 
Fig 1. The prescribing competency framework. The full competency framework can be found on the Royal Pharmaceutical Society website (www.rpharms.com/prescribingframework). Reproduced with permission from the Royal Pharmaceutical society.

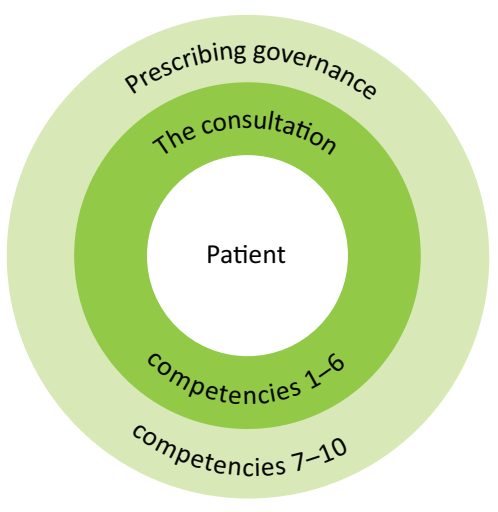

\section{The consultation}

1 Assess the patient 2 Consider the options 3 Reach a shared decision 4 Prescribe 5 Provide information 6 Monitor and review
Prescribing governance

7 Prescribe safely

8 Prescribe professionally 9 Improve prescribing practice 10 Prescribe as part of a team embedding of generic professional skills for all. The prescribing competency framework facilitates this, highlighting the importance to all healthcare providers of working within a team and a giving cognisance to the importance of wider systems.

Continued engagement between the Royal Pharmaceutical Society, the Academy of Medical Royal Colleges, the Royal College of Physicians, the Royal College of General Practitioners, the General Medical Council and Medical Schools Council to explore how the prescribing framework might be embedded in practice would be welcome.

\section{Conflicts of interest}

The authors declare no conflicts of interests.

\section{Acknowledgments}

The views expressed in this publication are those of the authors alone.

\section{References}

1 Duerden M, Avery T, Payne R. Polypharmacy and medicines optimisation. Making it safe and sound. London: The King's Fund, 2013.

2 Royal College of General Practitioners. Patient safety implications of general practice workload. London: RCGP, 2015.

3 Northern Ireland Audit Office. Primary care prescribing. Belfast: Northern Ireland Audit Office, 2014.

4 Future Hospital Commission. Future hospital: caring for medical patients. London: Royal College of Physicians, 2013.

5 Shape of training. Securing the future of excellent patient care. London: GMC, 2013.

6 Royal College of General Practitioners. The 2022 GP. A vision for general practice in the future NHS. London: RCGP, 2013.

7 NHS England. Clinical pharmacists in general practice pilot. Available online at www.england.nhs.uk/commissioning/primarycare-comm/gp-action-plan/cp-gp-pilot/ [Accessed 30 June 2016].

8 Royal Pharmaceutical Society. Medicines optimisation: helping patients make the most of their medicines. London: Royal Pharmaceutical Society, 2013.

9 Barber N, Parsons J, Clifford S, Darracott R, Horne R. Patients' problems with new medication for chronic conditions. Qual Saf Health Care 2004;13:172-5.

10 Barber ND, Alldred DP, Raynor DK et al. Care homes' use of medicines study: prevalence, causes and potential for harm of medication errors in care homes for older people. Qual Saf Health Care 2009; 18:341-6.

11 Cousins DH, Gerrett D, Warner B. A review of medication incidents reported to the National Reporting and Learning System in England and Wales over 6 years (2005-2010). Br J Clin Pharmacol 2012;74:597-604.
12 Dornan T, Ashcroft D, Heathfield $\mathrm{H}$ et al. An in depth investigation into causes of prescribing errors by foundation trainees in relation to their medical education. EQUIP study. Manchester: University of Manchester, 2010.

13 Avery T, Barber N, Ghaleb M et al. Investigating the prevalence and causes of prescribing errors in general practice: The PRACtICe study. Nottingham: University of Nottingham, 2012.

14 York Health Economics Consortium, The School of Pharmacy, University of London. Evaluation of the scale, causes and costs of waste medicines. York: University of York, 2010.

15 Pirmohamed M, James S, Meakin S et al. Adverse drug reactions as cause of admission to hospital: prospective analysis of 18820 patients. BMJ 2004;329:15-19.

16 National Institute for Health and Care Excellence. Medicines optimisation: the safe and effective use of medicines to enable the best possible outcomes. NICE guideline No 5. London: NICE, 2015.

17 Tomaszewski M, White C, Patel P et al. High rates of non-adherence to antihypertensive treatment revealed by high-performance liquid chromatography-tandem mass spectrometry (HP LC-MS/MS) urine analysis. Heart 2014;100:855-61.

18 Kohn LT, Corrigan JM, Donaldon MS (eds). To err is human: building a safer health system. Washington DC: National Academy Press, 2000.

19 Wilson T, Sheikh A. Enhancing public safety in primary care. BMJ 2002;324:584.

20 Howard RL, Avery AJ, Slavenburg S et al. Which drugs cause preventable admissions to hospital? A systematic review. $\mathrm{Br} \mathrm{J} \mathrm{Clin}$ Pharmacol 2007; 63:136-47.

21 NHS England. Five Year Forward View. NHS England, 2014.

22 NHS England. New care models - vanguard sites. Available online at www.england.nhs.uk/ourwork/futurenhs/new-care-models/ [Accessed 30 June 2016].

23 Royal Pharmaceutical Society. Professional standards for homecare services in England. London: Royal Pharmaceutical Society, 2013.

24 Ross S, Maxwell S. Prescribing and the core curriculum for tomorrow's doctors: BPS curriculum in clinical pharmacology and prescribing for medical students. Br J Clin Pharmacol 2012;74:644-61.

25 Prescribing Safety Assessment. Available online at https:// prescribingsafetyassessment.ac.uk/ [Accessed 30 June 2016].

26 Scottish Patient Safety Programme - Primary Care Shared Resources. Availale online at www.knowledge.scot.nhs.uk/spsp-pc/resourceslibrary/resource-detail.aspx?id=4070136 [Accessed 30 June 2016].

27 General Medical Council, Academy of Medical Royal Colleges. Developing a framework for generic professional capabilities. London: GMC, AoMRC, 2015.

28 Royal Pharmaceutical Society. A competency framework for all prescribers. London: Royal Pharmaceutical Society, 2016.

Address for correspondence: Dr C Picton, c/o Professional Support, The Royal Pharmaceutical Society, 66-68 East Smithfield, London E1W 1AW, UK.

Email: catherinepicton@t-online.de 